\title{
Trough instabilities in Boussinesq formulations for water waves
}

\section{Madsen, Per A.; Fuhrman, David R.}

Published in:

Journal of Fluid Mechanics

Link to article, DOI:

$10.1017 / \mathrm{jfm} .2020 .76$

Publication date:

2020

Document Version

Peer reviewed version

Link back to DTU Orbit

Citation (APA):

Madsen, P. A., \& Fuhrman, D. R. (2020). Trough instabilities in Boussinesq formulations for water waves. Journal of Fluid Mechanics, 889, [A38]. https://doi.org/10.1017/jfm.2020.76

\section{General rights}

Copyright and moral rights for the publications made accessible in the public portal are retained by the authors and/or other copyright owners and it is a condition of accessing publications that users recognise and abide by the legal requirements associated with these rights.

- Users may download and print one copy of any publication from the public portal for the purpose of private study or research.

- You may not further distribute the material or use it for any profit-making activity or commercial gain

- You may freely distribute the URL identifying the publication in the public portal

If you believe that this document breaches copyright please contact us providing details, and we will remove access to the work immediately and investigate your claim 


\title{
TROUGH INSTABILITIES IN BOUSSINESQ FORMULATIONS FOR WATER WAVES
}

\author{
Per A. Madsen and David R. Fuhrman \\ Dept. of Mechanical Engineering, Technical University of Denmark, \\ 2800 Kgs Lyngby, Denmark. Emall: PrM@Mek.Dtu.DK.
}

Version 09-09-2019

\section{Abstract}

Modern Boussinesq-type formulations for water waves typically incorporate fairly accurate linear dispersion relations and similar accuracy in nonlinear properties. This has extended their application range to higher values of $k h$ ( $k$ being wave number and $h$ the water depth) and it has allowed for a better representation of nonlinear irregular waves with a fairly large span of short waves and long waves. Unfortunately, we have often experienced a number of "mysterious" breakdowns or blowups, which have perplexed us for some time. A closer inspection has revealed that short-period noise can typically evolve in the deep troughs of wave trains in cases having relatively high spatial resolution. It appears that these potential "trough instabilities" have not previously been discussed in the literature. In the present work, we analyse this problem in connection with the fourth- and fifthorder Padé formulations by Agnon et al. (1999), the One-Step Padé and the Two-Step Taylor/Padé formulations by Madsen et al. (2002), and the multi-layer formulations by Liu et al. (2018). For completeness, we also analyse the popular, but older, formulations by Nwogu (1993) and Wei et al. (1995). We generally conclude, that trough instabilities may occur in any Boussinesq-type formulation incorporating nonlinear dispersive terms. This excludes most of the classical Boussinesq formulations, but includes all of the socalled "fully-nonlinear" formulations. Our instability analyses are successfully verified and confirmed by making simple numerical simulations of the same formulations implemented in 1D on a horizontal bottom. Furthermore, a remedy is proposed and tested on the One-Step and Two-Step formulations by Madsen et al. (2002). This demonstrates that the trough instabilities can be moved or removed by a relatively simple reformulation of the governing Boussinesq equations. Finally, we discuss the option of an implicit Taylor formulation combined with exact linear dispersion, which is the starting point for the explicit perturbation formulation by Dommermuth \& Yue (1987), i.e. the popular HOS (higher-order-spectral) formulations. In this case, we find no sign of trough instabilities.

KEY WORDS: Water waves, Boussinesq-type formulations, trough instabilities, Nyquist wave numbers, spectral simulations.

\section{INTRODUCTION}

Since the early 1990s, a number of alternative Boussinesq-type formulations have been derived in order to improve the range of applicability of linear and nonlinear properties embedded in the equations. In the beginning, the main focus was on the improvement of the embedded linear dispersion relation and this was achieved either by applying appropriate linear operators to the continuity and momentum equations (see e.g Madsen et al., 1991; Madsen \& Sørensen, 1992), by choosing appropriate velocity variables such as the horizontal velocity vector at mid-depth (see e.g. Nwogu, 1993), or by a combination of both techniques (see e.g. Madsen \& Schäffer, 1998). Later, developments focused 
on improving the embedded nonlinear properties such as the transfer functions for subharmonic and super-harmonic interactions, and this was typically achieved by allowing the nonlinear scaling parameter to be of order one, thus retaining nonlinear dispersive terms in the formulations (see e.g. Wei et al., 1995; Madsen \& Schäffer, 1998; Gobbi et al. 2000). In addition, some formulations also introduced a parameter-optimization of self-self interaction terms (see e.g. Kennedy et al. 2001; Lynett \& Liu, 2004). Despite these efforts, the general trend was that nonlinear properties were far less accurate than linear properties.

Agnon et al. (1999) were the first to present a Boussinesq formulation, which incorporated the same accuracy in nonlinear properties as in linear properties. Their procedure was based on an exact formulation of the boundary conditions at the free surface combined with an approximate solution to the Laplace equation given in terms of truncated Taylor series expansions, with variables being the vertical $\left(w_{0}\right)$ and horizontal $\left(u_{0}\right)$ velocity components defined at the expansion level $z_{0}=0$. A relatively high order connection between $w_{0}$ and $u_{0}$ was obtained by invoking a separate Padé enhancement of the kinematic bottom condition. Using a threshold of $2 \%$ error in the squared linear celerity, the resulting formulation was applicable up to $k h=6.2$ ( $k$ being the wave number and $h$ the water depth).

Madsen et al. (2002 \& 2003) generalized this procedure to expand with fifth order operators from an arbitrary vertical level $z_{E}$ (typically choosen to be the mid-depth, inspired by Nwogu, 1993) and by introducing pseudo-velocity variables $w_{E}$ and $u_{E}$ to achieve Padé approximations at the sea bottom as well as at the still water level. Using the threshold defined above, the resulting formulation was applicable up to $k h \simeq 26$. In addition, the formulation incorporated highly accurate velocity profiles accurate up to approximately $k h \simeq 12$ (analysed in detail by Agnon \& Madsen, 2003). Two different formulations were presented: a so-called Two-Step Taylor/Padé formulation with highly accurate nonlinear properties and a One-Step Padé formulation with slightly less accurate nonlinear properties.

Note that all Boussinesq formulations described above are based on a single-layer description expressed in terms of velocity variables $\left(w_{E}, u_{E}\right)$ or just $u_{E}$ defined at the expansion level $z_{E}$. Alternatively, Lynett \& Liu (2004) proposed a two-layer formulation with separate velocity polynomials within the layers and with additional interface conditions. The intention was to achieve relatively high accuracy, while allowing for lower-order operators in each layer. Using the threshold defined above, the resulting formulation was applicable up to $k h \simeq 6.8$. Later, two-layer models were also proposed by e.g. Chazel et al. (2009) and Liu \& Fang (2016). Recently, Liu et al. (2018) presented a multilayer approach which combines the techniques of Madsen et al. (2002) and Lynett \& Liu (2004): Within each layer a velocity profile is expanded from mid-depth of the layer $z_{n}$, and it is expressed in terms of the pseudo-velocity variables $w_{n}$ and $u_{n}$ to achieve Padé approximations at the sea bottom, at each of the interfaces and at the still water level. In addition to the exact boundary conditions at the free surface and at the sea bottom, interface conditions are added to secure continuity of $w_{n}$ and $u_{n}$. The formulation is very transparent and the method is highly accurate: Based on the threshold defined above, the formulations are applicable up to $k h \simeq 10,62,277$ and 938 for the one-layer, two-layer, three-layer and four-layer formulations invoking third-order operators within each layer.

From the very beginning, the motivation for improving linear and nonlinear properties in classical Boussinesq formulations has been to study the evolution and transformation of irregular wave trains over complex bathymetry. In general, this has indeed been 
achieved by the capacity incorporated in many of the formulations mentioned above. Unfortunately, now and then "mysterious" blow-ups occur and we have typically noticed that these blow-ups happen in connection with instabilities occuring in the troughs of the wave train whenever the Nyquist wave number is relatively high i.e. when the spatial resolution is relatively fine. This problem has perplexed the authors for some time. The present work presents a novel analysis of this problem in connection with a variety of the formulations mentioned above. Furthermore, we systematically verify the presence of analytical instabilities by making numerical spectral simulations under controlled conditions.

The exact equations for the water wave problem are specified in Section 3. The modern formulations by Agnon et al. (1999), Madsen et al. (2002), and Liu et al. (2018) are analysed and numerically checked in Sections 4, 5 and 6, respectively. Section 7 provides a new method to move or remove the trough instabilities. Older Boussinesq formulations are discussed in Section 8, and finally Section 9 discuss the option of Taylor expansions combined with exact linear dispersion relevant for the classical Higher Order Spectral methods (HOS). Summary and conclusions are given in Section 10.

\section{EXACT EQUATIONS FOR THE WATER WAVE PROBLEM}

A Cartesian coordinate system is adopted with the $x$-axis and $y$-axis located on the still-water plane and with the $z$-axis pointing vertically upwards. The fluid domain is generally bounded by the sea bed at $z=-h[x, y]$ and by the free surface at $z=\eta[x, y, t]$. Assuming irrotational flow, the velocity potential $\Phi$ is related to the velocity components by the definition

$$
\mathbf{u} \equiv \nabla \Phi, \quad w \equiv \Phi_{z},
$$

where $\nabla$ is the two-dimensional gradient operator defined by

$$
\nabla \equiv\left(\frac{\partial}{\partial x}, \frac{\partial}{\partial y}\right)
$$

Next, we introduce the surface variables $\mathbf{u}_{s} \equiv(\nabla \Phi)_{z=\eta}, w_{s} \equiv\left(\Phi_{z}\right)_{z=\eta}$ and $\Phi_{s} \equiv(\Phi)_{z=\eta}$ by which the exact kinematic surface condition reads

$$
\frac{\partial \eta}{\partial t}=w_{s}-\mathbf{u}_{s} \cdot \nabla \eta
$$

The exact dynamic surface condition can be expressed as

$$
\frac{\partial \Phi_{s}}{\partial t}=-g \eta-\frac{1}{2}\left(\nabla \boldsymbol{\Phi}_{s} \cdot \nabla \boldsymbol{\Phi}_{s}\right)+\frac{1}{2} w_{s}^{2}(1+\nabla \eta \cdot \nabla \eta)
$$

see e.g. Zakharov (1968) and Dommermuth \& Yue (1987). Alternatively, we may take the $\nabla$ gradient of (4), which leads to the velocity vector equation considered by e.g. Witting (1984), Agnon et al. (1999) and Madsen et al. (2002)

$$
\frac{\partial \mathbf{V}_{s}}{\partial t}=-g \nabla \eta-\nabla\left(\frac{1}{2} \mathbf{V}_{s} \cdot \mathbf{V}_{s}-\frac{1}{2} w_{s}^{2}(1+\nabla \eta \cdot \nabla \eta)\right)
$$

where

$$
\mathbf{V}_{s} \equiv \nabla \Phi_{s}=\mathbf{u}_{s}+w_{s} \nabla \eta
$$


Note that (3) together with either (4) or (5) define the fully nonlinear time-stepping problem. Within each time step, we need to establish a connection between the vertical and horizontal velocities at the free surface (the so-called Dirichlet-Neumann problem), and this requires that we solve the Laplace equation in the interior of the domain. An exact infinite series solution to this problem reads

$$
\begin{aligned}
\mathbf{u}[x, y, z, t] & =\sum_{n=0}^{\infty} \frac{(-1)^{n} z^{2 n}}{(2 n) !} \nabla^{2 n} \mathbf{u}_{0}+\sum_{n=0}^{\infty} \frac{(-1)^{n} z^{2 n+1}}{(2 n+1) !} \nabla^{2 n+1} w_{0} \\
w[x, y, z, t] & =\sum_{n=0}^{\infty} \frac{(-1)^{n} z^{2 n}}{(2 n) !} \nabla^{2 n} w_{0}-\sum_{n=0}^{\infty} \frac{(-1)^{n} z^{2 n+1}}{(2 n+1) !} \nabla^{2 n+1} \mathbf{u}_{0}, \\
\Phi[x, y, z, t] & =\sum_{n=0}^{\infty} \frac{(-1)^{n} z^{2 n}}{(2 n) !} \nabla^{2 n} \Phi_{0}+\sum_{n=0}^{\infty} \frac{(-1)^{n} z^{2 n+1}}{(2 n+1) !} \nabla^{2 n} w_{0}
\end{aligned}
$$

where $\left(\mathbf{u}_{0}, w_{0}\right)$ are defined at the still water level $z_{0}=0$ (see e.g. Madsen \& Schäffer, 1998; Agnon et al., 1999).

Thoughout the rest of this paper, we shall ignore motions in the $y$-direction and consider a constant water depth for simplicity. In this case, the kinematic bottom condition reads

$$
w_{b}=0, \quad \text { where } w_{b} \equiv\left(\Phi_{z}\right)_{z=-h},
$$

and $\nabla$ simplifies to

$$
\nabla \equiv \frac{\partial}{\partial x}
$$

\section{The formulation by Agnon, Madsen \& Schäffer (1999)}

4.1. The approximate solution to the Laplace equation. Agnon et al. (1999) truncated (7)-(9) at fifth order, by which the velocity field and the associated velocity potential simplified to

$$
\begin{aligned}
u[x, z, t] & =\left(1-\frac{z^{2}}{2} \nabla^{2}+\frac{z^{4}}{24} \nabla^{4}\right) u_{0}+\left(z \nabla-\frac{z^{3}}{6} \nabla^{3}+\frac{z^{5}}{120} \nabla^{5}\right) w_{0}, \\
w[x, z, t] & =\left(1-\frac{z^{2}}{2} \nabla^{2}+\frac{z^{4}}{24} \nabla^{4}\right) w_{0}-\left(z \nabla-\frac{z^{3}}{6} \nabla^{3}+\frac{z^{5}}{120} \nabla^{5}\right) u_{0}, \\
\Phi[x, z, t] & =\left(1-\frac{z^{2}}{2} \nabla^{2}+\frac{z^{4}}{24} \nabla^{4}\right) \Phi_{0}+\left(z-\frac{z^{3}}{6} \nabla^{2}+\frac{z^{5}}{120} \nabla^{4}\right) w_{0} .
\end{aligned}
$$

In order to satisfy the kinematic bottom condition (10), they utilized (13) with $z=-h$, but Padé enhanced the resulting equation to obtain

$$
\left(1-\frac{4}{9} h^{2} \nabla^{2}+\frac{1}{63} h^{4} \nabla^{4}\right) w_{0}+\left(h \nabla-\frac{1}{9} h^{3} \nabla^{3}+\frac{1}{945} h^{5} \nabla^{5}\right) u_{0}=0 .
$$


Finally, the connection to the surface variables was established by using (12)-(14) with $z=\eta$, leading to

$$
\begin{aligned}
& u_{s}=\left(1-\frac{1}{2} \eta^{2} \nabla^{2}+\frac{1}{24} \eta^{4} \nabla^{4}\right) u_{0}+\left(\eta \nabla-\frac{1}{6} \eta^{3} \nabla^{3}+\frac{1}{120} \eta^{5} \nabla^{5}\right) w_{0}, \\
& w_{s}=\left(1-\frac{1}{2} \eta^{2} \nabla^{2}+\frac{1}{24} \eta^{4} \nabla^{4}\right) w_{0}-\left(\eta \nabla-\frac{1}{6} \eta^{3} \nabla^{3}+\frac{1}{120} \eta^{5} \nabla^{5}\right) u_{0}, \\
& \Phi_{s}=\left(1-\frac{1}{2} \eta^{2} \nabla^{2}+\frac{1}{24} \eta^{4} \nabla^{4}\right) \Phi_{0}+\left(\eta-\frac{1}{6} \eta^{3} \nabla^{2}+\frac{1}{120} \eta^{5} \nabla^{4}\right) w_{0} .
\end{aligned}
$$

It should be noted, that Agnon et al. (1999) actually only considered a fourth-order connection in (16)-(18), i.e. they did not include the terms proportional to $\nabla^{5}$. In the following, however, we shall analyze both the fifth-order and the fourth-order formulations.

4.2. Analysis of the embedded linear dispersion relation. The linear dispersion relation embedded in the formulation by Agnon et al. (1999) is analyzed by looking for harmonic solutions on the form

$$
\eta=\varepsilon A_{0} \cos [k x-\omega t], \quad u_{0}=\varepsilon B_{0} \cos [k x-\omega t], \quad w_{0}=\varepsilon C_{0} \sin [k x-\omega t],
$$

where $\varepsilon \ll 1$. First of all, (15) directly leads to the connection

$$
C_{0}=B_{0} F_{0}[\kappa],
$$

with

$$
F_{0}[\kappa] \equiv \kappa\left(\frac{1+\frac{1}{9} \kappa^{2}+\frac{1}{945} \kappa^{4}}{1+\frac{4}{9} \kappa^{2}+\frac{1}{63} \kappa^{4}}\right), \quad \text { and } \kappa \equiv k h .
$$

Second, by inserting (19) into (16)-(17) and collecting terms of order $O(\varepsilon)$, we obtain the leading order approximations $u_{s} \simeq u_{0}, w_{s} \simeq w_{0}$, while (6) leads to $V_{s} \simeq u_{0}$. Consequently (3) and (5) lead to the homogeneous problem

$$
\left(\begin{array}{cc}
\omega m_{11} & m_{12} \\
m_{21} & \omega m_{22}
\end{array}\right)\left(\begin{array}{c}
A_{0} \\
B_{0}
\end{array}\right)=\left(\begin{array}{l}
0 \\
0
\end{array}\right),
$$

with

$$
m_{11}=1, \quad m_{12}=-F_{0}[\kappa], \quad m_{21}=-g k, \quad m_{22}=1 .
$$

The homogeneous problem requires the determinant of the matrix in (22) to be zero, and this leads to the dispersion relation

$$
\frac{c^{2}}{g h} \equiv \frac{\omega^{2}}{k^{2} g h}=\frac{F_{0}[\kappa]}{\kappa} .
$$

Using a threshold of $2 \%$ error compared to the fully dispersive target, (24) turns out to be applicable up to $k h=6.2$.

4.3. Numerical implementation on a constant depth. From our experience with modelling nonlinear regular and irregular waves, it appears that "mysterious" numerical blow-ups typically occur in relatively deep troughs of the overall surface elevation whenever the Nyquist wave number is high. In order to demonstrate this problem, we 
first implement a spectral model solving the equations by Agnon et al. (1999) on a constant depth. The time stepping of (3)-(4) is based on a fourth-order Runge-Kutta method, and all spatial derivatives are handled by spectral representations involving a toggling from physical space to Fourier space and back into physical space by using Fast Fourier Transforms. We represent the solution in $k$-space by considering $N$ discrete wave numbers $\left(k_{j}=j \triangle k\right.$, with $\left.j=1,2, \ldots, N\right)$ and $N_{x}=2 N$ grid points $\left(x_{i}=i \triangle x\right.$, with $\left.i=1,2, \ldots, N_{x}\right)$. The Nyquist wave number is determined by $k_{N}=\pi / \triangle x$.

Within each of the four sub-time steps in the Runge-Kutta procedure, we need to determine $w_{s}$ on the basis of $\left(\eta, \Phi_{s}\right)$ known at all grid points. First, $\left(\Phi_{0}, u_{0}, w_{0}\right)$ are described by

$$
\begin{aligned}
& \Phi_{0}[x]=a_{0}+\sum_{j=1}^{N}\left(a_{j} \sin \left[k_{j} x\right]-b_{j} \cos \left[k_{j} x\right]\right), \\
& u_{0}[x]=\sum_{j=1}^{N} k_{j}\left(a_{j} \cos \left[k_{j} x\right]+b_{j} \sin \left[k_{j} x\right]\right), \\
& w_{0}[x]=\sum_{j=1}^{N} k_{j} F_{0}\left[k_{j} h\right]\left(a_{j} \sin \left[k_{j} x\right]-b_{j} \cos \left[k_{j} x\right]\right),
\end{aligned}
$$

with $a_{N} \equiv 0$. Second, (18) is satisfied in each grid point, which leads to $2 N$ linear equations with the unknowns $a_{j}$ and $b_{j}$ for $j=1,2, \ldots, N$. This problem is solved in Mathematica by invoking the linear matrix solver LinearSolve. Finally, $w_{s}$ is determined using (17).

With the numerical model at hand, we first make a simulation of a standing wave problem defined by the initial conditions

$$
\eta / h=\mu \cos \left[k_{1} x\right], \text { and } \Phi_{s}=0,
$$

with amplitude $\mu=0.05$ and water depth $h=1 \mathrm{~m}$. The spectral and numerical resolution for this simulation is chosen as

$$
N=10, k_{1} h=1.0, k_{N} h=10, N_{x}=20, \triangle x=\pi / 10 \mathrm{~m},
$$

with the time step $\triangle t=0.04 \mathrm{~s}$ corresponding to a Courant number $C_{r} \equiv \triangle t \sqrt{g h} / \triangle x=$ 0.4. This simulations runs for 500 time steps (approximately ten wave periods) without showing any signs of instability.

Then, to illustrate the problem of trough instabilities, we maintain the same initial conditions as above, but change the spectral and numerical resolution to

$$
N=40, k_{1} h=1.0, k_{N} h=40, N_{x}=80, \triangle x=\pi / 40 \mathrm{~m},
$$

with the time step of $\triangle t=0.01 \mathrm{~s}$ leading again to $C_{r}=0.4$. This time the simulation starts to go unstable after approximately 89 time steps (shown as the black curve in Figure 1). It is obvious that the instability evolves from the trough of the wave. After 95 time steps (shown as the red curve in Figure 1), the instability has expanded to the complete profile and soon after the simulation blows up. It appears that the value of $k_{N} h$ is important for the instability just observed.

In order to study the trough instability in more detail, we modify the initial conditions to

$$
\eta / h=\delta+\mu \cos \left[k_{1} x\right], \text { and } \Phi_{s}=0
$$


where $\delta=-0.05, \mu=0.005$ and $h=1 \mathrm{~m}$. This represents a perturbation, having amplitude ten times smaller than the previous wave and which runs with a negative offset $\delta$ equal to the trough of the previous wave. Again the numerical setup is given by (30). As shown in Figure 2, this simulation starts to go unstable after approximately 60 time steps. It is, however, possible to spot the growing instability at a much earlier stage by monitoring the complex $k$-spectrum of the surface elevation (shown in Figure 3 after 40 time steps). The horizontal axis is $k_{j} h$ with $j=1,2, \ldots, N$. The spectrum shows very clearly that an instability is evolving at $k_{j} h=18$. With this procedure, we are now able to detect the critical wave numbers for any value of $\delta$. This will be utilized in the following sections.

4.4. Analysis of trough instabilities. A simple analysis of the trough instability illustrated in Figures 1-3 can be conducted by modifying (19) to

$$
\eta=\delta h+\varepsilon A_{0} \cos [k x-\omega t], \quad u_{0}=\varepsilon B_{0} \cos [k x-\omega t], \quad w_{0}=\varepsilon C_{0} \sin [k x-\omega t],
$$

where $\delta h$ represents the trough of the overall wave train, while the harmonic variation represents a short wave disturbance with small nonlinearity i.e. $\varepsilon \ll 1$. The offset is assumed to be order $O(1)$ and we consider the interval $-1 \leq \delta \leq 0$. On this basis, we shall conduct an analysis to order $O(\varepsilon)$ of the nonlinear governing equations.

Note that by inserting (32) into (16)-(18) and collecting terms of order $O(\varepsilon)$, all powers of $\eta$ will contribute to the $O(\varepsilon)$-terms. As a consequence we obtain a homogeneous problem similar to (22), but with different $m_{12}$ and $m_{22}$ coefficients defined by

$$
\begin{aligned}
& m_{12}=-F_{0}[\kappa]\left(1+\frac{1}{2} \delta^{2} \kappa^{2}+\frac{1}{24} \delta^{4} \kappa^{4}\right)-\left(\delta \kappa+\frac{1}{6} \delta^{3} \kappa^{3}+\frac{1}{120} \delta^{5} \kappa^{5}\right), \\
& m_{22}=\left(1+\frac{1}{2} \delta^{2} \kappa^{2}+\frac{1}{24} \delta^{4} \kappa^{4}\right)+F_{0}[\kappa]\left(\delta \kappa+\frac{1}{6} \delta^{3} \kappa^{3}+\frac{1}{120} \delta^{5} \kappa^{5}\right) .
\end{aligned}
$$

As mentioned earlier, Agnon et al. (1999) used a fourth-order rather than a fifth-order connection in (16)-(18), which implies that the $\kappa^{5}$-terms in (33)-(34) vanish.

First, we focus directly on the resulting expression for the linear celerity, which is again determined from the determinant of the matrix in (22). It turns out that it is now prone to singularities for specific values of $\delta$. We determine $\delta$ as a function of $\kappa$ for which the inverse of the celerity goes to zero, and the solutions are shown as the full (orange) lines in Figure 4 (for the fourth-order connection) and in Figure 5 (for the fifth-order connection).

Second, we focus on a stability analysis in terms of the variables $\eta$ and $u_{0}$. This can be formulated as the following algebraic problem

$$
\left(\begin{array}{c}
\omega A_{0} \\
\omega B_{0}
\end{array}\right)=\left(\begin{array}{ll}
n_{11} & n_{12} \\
n_{21} & n_{22}
\end{array}\right)\left(\begin{array}{c}
A_{0} \\
B_{0}
\end{array}\right),
$$

where the left hand side represents the time derivatives of $\eta$ and $u_{0}$. Instabilities will occur if the $n$-matrix contains eigenvalues with imaginary parts. It is straight forward to rewrite (22) to the form of (35) and we then obtain

$$
n_{11}=0, \quad n_{12}=-\frac{m_{12}}{m_{11}}, \quad n_{22}=0, \quad n_{21}=-\frac{m_{21}}{m_{22}} .
$$

The $\delta$-regions associated with imaginary eigenvalues are shown as the gray areas in Figures 4 and 5. Within these areas instabilities can occur, but the full lines determined 
from the zeros of the inverse celerities indicate the strongest growth rates. The trend is that the trouble zone gets closer and closer to the still water level $(z=0)$ for increasing values of $k h$. In this connection it should be emphasized that for a given numerical simulation, it is the Nyquist wave number that really matters, since all resolved wave numbers must be stable to avoid instability. This will typically be orders of magnitude larger than the wave numbers representing the physical wave train at hand, since accurate simulations require good spatial resolution.

In order to verify and confirm the validity of the stablity analysis, we again run the spectral model discussed in the previous section. The setup follows (31) with (30), and we generally use $\mu=0.005$ while varying the offset $\delta$. In each simulation, the $k$-spectrum of $\eta$ is monitored, and this clearly indicates if the simulation is stable, or unstable and at which wave number the instability is provoked. Figures 4 and 5 include the numerical results presented in the following way: 1) A stable simulation is illustrated by an open circle, and for this case we choose to locate the open markers at the Nyquist wave number; 2) A simulation which blows up or which has a significant growth occuring at some of the discrete wave numbers is illustrated by filled circles. These are located at the discrete wave number showing the largest growth (e.g. $k_{j} h=18$ as seen in Figure 3). We notice from Figures 4 and 5 , that these markers generally fall on the theoretical curves representing the strongest growth rate in the instability. Hence, there is generally a very good agreement between the theory and the numerical simulations.

From the investigations presented in this section, we conclude that if the deepest trough of the free surface $\left(\eta_{\min } / h\right)$ and the spatial resolution $\left(k_{N} h\right)$ are such that they fall within the unstable (gray) regions (e.g. Figures 4 and 5), the numerical model will be prone to trough instabilities. This result is independent of time step, time stepping scheme and the numerical method used to approximate spatial derivatives.

5. The One-Step and Two-Step formulations by Madsen et Al. (2002)

5.1. The approximate solutions to the Laplace equation. In this section, we consider the Boussinesq formulations developed by Madsen et al. (2002). We focus on the fifth-order formulations expressed in a single horizontal dimension. First of all, the following Padé enhanced velocity formulation is applied

$$
\begin{aligned}
u[x, z, t] & =\left(1+\alpha_{2}[z] \nabla^{2}+\alpha_{4}[z] \nabla^{4}\right) u_{E}+\left(\beta_{1}[z] \nabla+\beta_{3}[z] \nabla^{3}+\beta_{5}[z] \nabla^{5}\right) w_{E}, \\
w[x, z, t] & =\left(1+\alpha_{2}[z] \nabla^{2}+\alpha_{4}[z] \nabla^{4}\right) w_{E}-\left(\beta_{1}[z] \nabla+\beta_{3}[z] \nabla^{3}+\beta_{5}[z] \nabla^{5}\right) u_{E}, \\
\Phi[x, z, t] & =\left(1+\alpha_{2}[z] \nabla^{2}+\alpha_{4}[z] \nabla^{4}\right) \Phi_{E}+\left(\beta_{1}[z]+\beta_{3}[z] \nabla^{2}+\beta_{5}[z] \nabla^{4}\right) w_{E},
\end{aligned}
$$

where $\left(u_{E}, w_{E}, \Phi_{E}\right)$ are pseudo-variables defined at the expansion level $z_{E}=-h / 2$ and where the $\alpha-$ and $\beta$-coefficients are defined by

$$
\begin{aligned}
& \alpha_{2}[z]=-\left(\frac{\left(z-z_{E}\right)^{2}}{2}-\frac{z_{E}^{2}}{18}\right) \\
& \alpha_{4}[z]=\left(\frac{\left(z-z_{E}\right)^{4}}{24}-\frac{z_{E}^{2}\left(z-z_{E}\right)^{2}}{36}+\frac{z_{E}^{4}}{504}\right), \\
& \beta_{1}[z]=\left(z-z_{E}\right), \\
& \beta_{3}[z]=-\left(\frac{\left(z-z_{E}\right)^{3}}{6}-\frac{z_{E}^{2}\left(z-z_{E}\right)}{18}\right) \\
& \beta_{5}[z]=\left(\frac{\left(z-z_{E}\right)^{5}}{120}-\frac{z_{E}^{2}\left(z-z_{E}\right)^{3}}{108}+\frac{z_{E}^{4}\left(z-z_{E}\right)}{504}\right) .
\end{aligned}
$$


This velocity field is far more accurate than the one proposed by Agnon et al. (1999), but it should be mentioned that (37)-(44) only provides genuine Padé properties at the sea bottom $z=-h$ and at the linearized free surface $z=0$. Madsen \& Agnon (2003) derived a more advanced velocity formulation with the objective of achieving Padé velocity properties throughout the interval $-h \leq z \leq 0$, but without improving the fundamental linear and nonlinear properties of the system.

Madsen et al. (2002) considered two different formulations: A so-called One-Step Padé formulation where (37)-(44) are applied within the entire water body $-h \leq z \leq \eta$, and a so-called Two-Step Taylor/Padé formulation where (37)-(44) are applied within the interval $-h \leq z \leq 0$, while (12)-(14) are applied within the dynamic top region $0 \leq z \leq \eta$.

In both methods, the kinematic bottom condition (on a constant depth) reads

$$
\left(1-\frac{1}{9} h^{2} \nabla^{2}+\frac{1}{1008} h^{4} \nabla^{4}\right) w_{E}+\left(\frac{1}{2} h \nabla-\frac{1}{72} h^{3} \nabla^{3}+\frac{1}{30240} h^{5} \nabla^{5}\right) u_{E}=0,
$$

which is obtained by using $z=-h$ in (40)-(44), while requiring that $w[x,-h, t]=0$.

For the Two-Step method, it is also relevant to determine the still water variables by utilizing (37)-(44) with $z=0$, which lead to

$$
\begin{aligned}
& u_{0}=\left(1-\frac{1}{9} h^{2} \nabla^{2}+\frac{1}{1008} h^{4} \nabla^{4}\right) u_{E}+\left(\frac{1}{2} h \nabla-\frac{1}{72} h^{3} \nabla^{3}+\frac{1}{30240} h^{5} \nabla^{5}\right) w_{E},(46) \\
& w_{0}=\left(1-\frac{1}{9} h^{2} \nabla^{2}+\frac{1}{1008} h^{4} \nabla^{4}\right) w_{E}-\left(\frac{1}{2} h \nabla-\frac{1}{72} h^{3} \nabla^{3}+\frac{1}{30240} h^{5} \nabla^{5}\right) u_{E} .(47)
\end{aligned}
$$

These expressions are then combined with (12)-(14) to provide the surface variables. In contrast, the surface variables in the One-Step method are obtained directly from (37)-(44) with $z=\eta$.

5.2. Analysis of the embedded linear dispersion relation. The linear properties of the Two-Step and One-Step formulations are identical. Again, we follow the procedure described in section 4.2 and look for harmonic solutions on the form of (19). Additionally, we describe the pseudo-velocities by

$$
u_{E}=\varepsilon B_{1} \cos [k x-\omega t], \quad w_{E}=\varepsilon C_{1} \sin [k x-\omega t] .
$$

Now, (45) directly leads to the connection

$$
C_{1}=B_{1} F_{1}[\lambda]
$$

with

$$
F_{1}[\lambda] \equiv \lambda\left(\frac{1+\frac{1}{9} \lambda^{2}+\frac{1}{945} \lambda^{4}}{1+\frac{4}{9} \lambda^{2}+\frac{1}{63} \lambda^{4}}\right), \quad \text { and } \lambda \equiv k\left(h+z_{E}\right)=\frac{1}{2} \kappa .
$$

Next, we utilize (46)-(47) to establish the connection

$$
C_{0}=B_{0} F_{0}[\kappa],
$$

where

$$
F_{0}[\kappa]=\kappa\left(\frac{1+\frac{5}{36} \kappa^{2}+\frac{47}{11340} \kappa^{4}+\frac{19}{544320} \kappa^{6}+\frac{1}{15240960} \kappa^{8}}{1+\frac{17}{36} \kappa^{2}+\frac{16}{567} \kappa^{4}+\frac{1}{2240} \kappa^{6}+\frac{29}{15240960} \kappa^{8}+\frac{1}{914457600} \kappa^{10}}\right) .
$$

The resulting linear celerity is again given by (24) which is now combined with (52). As a result, we conclude that the linear dispersion relation is applicable up to $k h=25.8$ based on a threshold of $2 \%$ error compared to the fully dispersive target. 
5.3. Instability analysis of the Two-Step method and its numerical verification. Having established the spectral connection between $w_{0}$ and $u_{0}$ in terms of (51)(52), the instability analysis of the Two-Step Taylor/Padé formulation now follows the procedure from section 4.3 very closely. As a result, we again obtain $(22)$ with $m_{11}=1$ and $m_{21}=-g k$, while $\left(m_{12}, m_{22}\right)$ are given by (33)-(34) with $F_{0}[\kappa]$ determined by $(52)$. The stability analysis leads to (35) with $\left(n_{11}, n_{12}, n_{21}, n_{22}\right)$ defined by (36).

The numerical implementation of the Two-Step method is done in the following way: As described in section 4.4, we time step (3)-(4) by the fourth-order Runge-Kutta method. Within each of the four sub-time steps, we need to determine $w_{s}$ on the basis of $\left(\eta, \Phi_{s}\right)$ known in all grid points. Typically, this procedure would involve the variables $\left(u_{E}\right.$, $\left.w_{E}\right)$ as well as $\left(\Phi_{0}, u_{0}, w_{0}\right)$ and equations (12)-(14) and (46)-(45). However, by solving the problem in the spectral domain, we can utilize the connection between $u_{0}$ and $w_{0}$ established in (51)-(52). With this shortcut, the implementation is fundamentally identical to what was described in section 4.4 , and all we need to do is to insert the new expression for $F_{0}[\kappa]$ defined by $(52)$.

Figure 6 shows the instability analysis compared to the numerical simulations. Again, the $\delta$-regions associated with imaginary eigenvalues are shown as the gray areas, while the full lines represent the zeros of the inverse celerities and define the strongest growth rates. The open circles indicate that the numerical simulation is stable with the marker placed at the Nyquist wave number, while the filled circles indicate that a significant growth takes place at the wave number $k h$ corresponding to the location of this marker. With the chosen Nyquist wave number of $k_{N} h=40$, solutions are stable for $\delta \geq-0.03$ and unstable within the interval of $-0.12 \leq \delta \leq-0.04$. Within the interval $-0.07 \leq \delta \leq-0.04$, the instabilities occur at the Nyquist wave number, but within $-0.12 \leq \delta \leq-0.08$ instabilities occur at smaller wave numbers, and the markers can be seen to follow the full line representing the strongest growth rates. Finally, we notice that for $\delta \leq-0.13$, all simulations are again stable. The reason is that the line of instability is very thin in this region, and it has not been triggered with the chosen discrete representation of wave numbers.

5.4. Instability analysis of the One-Step method and its numerical verification. The instability analysis of the One-Step formulation will deviate from the TwoStep analysis, as we now have to expand directly from $z_{E}$ to $z=\eta$ using (37)-(44). As a result, we formally obtain $(22)$ with $m_{11}=1$ and $m_{21}=-g k$, but with

$$
\begin{aligned}
& m_{12}=-F_{1}\left[\frac{\kappa}{2}\right]\left(1-k^{2} \alpha_{2}[\delta h]+k^{4} \alpha_{4}[\delta h]\right)-\left(k \beta_{1}[\delta h]-k^{3} \beta_{3}[\delta h]+k^{5} \beta_{5}[\delta h]\right) \\
& m_{22}=\left(1-k^{2} \alpha_{2}[\delta h]+k^{4} \alpha_{4}[\delta h]\right)+F_{1}\left[\frac{\kappa}{2}\right]\left(k \beta_{1}[\delta h]-k^{3} \beta_{3}[\delta h]+k^{5} \beta_{5}[\delta h]\right)
\end{aligned}
$$

The stability analysis again formally leads to (35) with $\left(n_{11}, n_{12}, n_{21}, n_{22}\right)$ defined by (36).

The numerical implementation of the One-Step method is slightly different from the previous two cases: Within each of the four sub-time steps, we need to determine $w_{s}$ on the basis of $\left(\eta, \Phi_{s}\right)$ known in all grid points and this procedure now involves the variables $\left(\Phi_{E}\right.$, $\left.u_{E}, w_{E}\right)$ and equations (37)-(44). In this procedure, we utilize the connection between $u_{E}$ and $w_{E}$ established in (49)-(50).

Figure 7 shows the instability analysis compared to the numerical simulations. The pattern is obviously quite different from Figure 5 . With the chosen Nyquist wave number of $k_{N} h=40$, solutions are stable for $\delta \geq-0.06$. Instabilities show up within the intervals 
of $-0.13 \leq \delta \leq-0.07$ and $-0.27 \leq \delta \leq-0.24$ and they typically follow the lines of strongest growth rate. Within the interval $-0.23 \leq \delta \leq-0.14$, most simulations are stable, again because the line of instability is very thin in this region. Only for the case of $\delta=-0.17$, the instability line has been triggered with the chosen discrete representation of wave numbers. It should be mentioned, that more instability regions appear within the interval $-1 \leq \delta \leq-0.35$ (not shown in Figure 7 ).

\section{The multi-layer formulations by LiU et Al. (2018)}

6.1. The approximate solutions to the Laplace equation. Liu et al. (2018) presented a new multi-layer approach which basicly extended the techniques of Madsen et al. (2002) to multiple layers. The formulation was valid on a mildly sloping topography, but in the following we shall summarize and analyse it on a constant depth. Within each layer $i=1,2, \ldots, M$, a velocity profile is expanded from mid-depth of the layer $z_{i}$ and it is expressed in terms of the pseudo-velocity variables $w_{i}$ and $u_{i}$ to achieve Padé approximations at the sea bottom, at each of the interfaces and at the still water level. Following Liu et al. (2018) only third-order operators will be invoked, but it is straight forward to increase the order of these operators.

Within each layer the bottom velocities $\left(u_{i}^{-}, w_{i}^{-}\right)$and the surface velocities $\left(u_{i}^{+}, w_{i}^{+}\right)$ are given by

$$
\begin{aligned}
& u_{i}^{ \pm}=\left(1-\frac{2}{5}\left(\gamma_{i} h\right)^{2} \nabla^{2}\right) u_{i} \pm\left(\gamma_{i} h \nabla-\frac{1}{15}\left(\gamma_{i} h\right)^{3} \nabla^{3}\right) w_{i} \\
& w_{i}^{ \pm}=\left(1-\frac{2}{5}\left(\gamma_{i} h\right)^{2} \nabla^{2}\right) w_{i} \mp\left(\gamma_{i} h \nabla-\frac{1}{15}\left(\gamma_{i} h\right)^{3} \nabla^{3}\right) u_{i},
\end{aligned}
$$

where $\gamma_{i}$ are parameters calibrated to obtain optimal performance of the system. Horizontal and vertical velocities are matched at the interfaces between the layers i.e.

$$
u_{i-1}^{-}=u_{i}^{+}, \text {and } w_{i-1}^{-}=w_{i}^{+} \text {for } i=2, \ldots, M
$$

Similarly, the condition at the still water level $z=0$ reads

$$
u_{0}=u_{1}^{+}, \text {and } w_{0}=w_{1}^{+},
$$

while the kinematic bottom condition reads

$$
w_{M}^{-}=0
$$

At the free surface, the exact kinematic and dynamic surface conditions are invoked i.e. (3) with (4) or (5). Finally, the free surface variables are connected to the variables at $z=0$ by (16)-(18) but without the fifth- and fourth-order operators.

6.2. Analysis of the embedded linear dispersion relations. The linear analysis of the multi-layer system follows section 4.4 quite closely i.e. we start by assuming harmonic solutions on the form

$$
u_{j}=\varepsilon B_{j} \cos [k x-\omega t], \quad w_{j}=\varepsilon C_{j} \sin [k x-\omega t] \text { for } j=1,2, \ldots N .
$$

First, we utilize (59) to establish the connection

$$
C_{M}=B_{M} F_{M}[\kappa]
$$


with

$$
F_{M}[\lambda] \equiv \lambda\left(\frac{1+\frac{1}{15} \lambda^{2}}{1+\frac{2}{5} \lambda^{2}}\right), \quad \text { where } \lambda \equiv \gamma_{M} k h .
$$

Next, by considering each of the interface conditions $(57)$, we determine $\left(B_{i-1}, C_{i-1}\right)$ in terms of $\left(B_{i}, C_{i}\right)$. Finally from (58), we determine $\left(B_{0}, C_{0}\right)$ in terms of $\left(B_{1}, C_{1}\right)$ and obtain

$$
C_{0}=B_{0} F_{0}[\kappa],
$$

which leads to the linear celerity in combination with (24). Obviously, the complexity and accuracy of $F_{0}[\kappa]$ quickly increases with the number of layers.

The one-layer formulation is actually similar to the Two-Step Taylor/Padé formulation by Madsen et al. (2002) except that third-order operators are applied instead of fifthorder operators. We note that for this special case (57) is not invoked. Liu et al. (2018) use the optimized coefficient $\gamma_{1}=\frac{1}{2}$ and as a result of the linear analysis we obtain

$$
F_{0}[\kappa]=\frac{\kappa\left(1+\frac{7}{60} \kappa^{2}+\frac{1}{600} \kappa^{4}\right)}{\left(1+\frac{9}{20} \kappa^{2}+\frac{11}{600} \kappa^{4}+\frac{1}{14400} \kappa^{6}\right)} .
$$

As a result, we conclude that the linear dispersion relation is applicable up to $k h=10$ based on a threshold of $2 \%$ error compared to the fully dispersive target.

In case of the two-layer formulation, the numerator and denominator of $F_{0}[\kappa] / \kappa$ are $10 t h$-order and $12 t h$-order polynomials in $\kappa$. For this case, Liu et al. (2018) used the optimized coefficients

$$
\gamma_{1}=0.1053, \quad \gamma_{2}=0.3947
$$

by which the celerity becomes applicable up to $k h=62$.

In case of the three-layer formulation, the numerator and denominator of $F_{0}[\kappa] / \kappa$ are $16 t h$-order and 18th-order polynomials in $\kappa$. For this case, Liu et al. (2018) used the optimized coefficients

$$
\gamma_{1}=0.025, \quad \gamma_{2}=0.091, \quad \gamma_{3}=0.384,
$$

by which the celerity becomes applicable up to $k h=277$.

Finally, in case of the four-layer formulation, the numerator and denominator of $F_{0}[\kappa] / \kappa$ are $22 t h$-order and $24 t h$-order polynomials in $\kappa$. For this case, Liu et al. (2018) used the optimized coefficients

$$
\gamma_{1}=0.008, \quad \gamma_{2}=0.023, \quad \gamma_{3}=0.103, \quad \gamma_{4}=0.366,
$$

by which the celerity becomes applicable up to $k h=938$.

6.3. Instability analysis and its numerical verification. The one-, two-, threeand four-layer formulations by Liu et al. (2018) can all be analysed and numerically implemented like the method of Agnon et al. (1999) described in sections 4.3-4.4. All we need to do is to apply the relevant solution for $F_{0}[\kappa]$ describing the spectral connection between $w_{0}$ and $u_{0}$.

Figure 8 shows the instability analysis for the one-layer method compared to the numerical simulations. Obviously, this pattern is similar to the one from Figure 5 but the instability zone is larger due to the less accurate linear dispersion relation. With the Nyquist wave number at $k_{N} h=40$, the method is stable for $\delta \geq-0.01$, while 
instabilities systematically occur within $-0.16 \leq \delta \leq-0.02$. Below this limit, the zone of instability becomes very narrow and as a consequence instabilities are more sporadic: Stable numerical solutions are found for $\delta=-0.17,-0.19$ and -0.21 , while unstable solutions are found for $\delta=-0.18$ and -0.20 .

Figure 9 shows the instability analysis for the two-layer method compared to the numerical simulations. Notice, that with the much more accurate dispersion relation, the zone of instabilities has moved to much higher values of $k h$ and consequently we have increased the Nyquist wave number in the numerical model to $k_{N} h=200$. We notice that the method is stable for $\delta \geq-0.003$, while instabilities systematically occur within $-0.03 \leq \delta \leq-0.004$. Below this limit, the zone of instability becomes very narrow and as a consequence most of the simulations are stable (exept for the case of $\delta=-0.04$ ).

Figure 10 shows the instability analysis for the three-layer method. Due to the significant improvement of the accuracy of the dispersion relation, the zone of potential instabilities has now moved to $k h>200$. Within the interval of $200 \leq k h \leq 400$, the instability region falls in $-0.008 \leq \delta \leq-0.004$ i.e. it is extremely thin. Naturally this trend is further extended when considering the four-layer method as shown in Figure 11. Now the zone of instability has now moved to $k h>700$ and within the interval of $700 \leq k h \leq 1200$, the instability region falls in $-0.0038 \leq \delta \leq-0.001$.

\section{Removing instabilities in the formulations by Madsen et AL. (2002)}

In this section we provide a method which can remove or move the instabilities in the various Boussinesq-type formulations discussed in the previous sections. We shall demonstrate the method on the Two-Step Taylor/Padé formulation by Madsen et al. (2002), but we emphasize that it is effective for all the formulations discussed in sections 4-6.

7.1. The approximate solution to the Laplace equation. In the following we generalize the Two-Step Taylor/Padé formulation by Madsen et al. (2002) with the objective of removing the instability problems discussed in section 5 . The first step is to use a flexible top level $z_{0}$ instead of using the conventional still water level $z_{0}=0$. This calls for a generalization of the upper Taylor formulation (12)-(14), which will be expanded from $z_{0}$. At the same time, the lower Padé formulation (37)-(44) needs to be generalized so that it now provides Padé properties at $z_{0}$ as well as at the sea bottom. Both velocity formulations can be combined into the following fifth-order formulation

$$
\begin{aligned}
u[x, z, t] & =\left(1+\alpha_{2}^{ \pm}[z] \nabla^{2}+\alpha_{4}^{ \pm}[z] \nabla^{4}\right) u^{ \pm}+\left(\beta_{1}^{ \pm} \nabla+\beta_{3}^{ \pm}[z] \nabla^{3}+\beta_{5}^{ \pm}[z] \nabla^{5}\right) w^{ \pm}, \\
w[x, z, t] & =\left(1+\alpha_{2}^{ \pm}[z] \nabla^{2}+\alpha_{4}^{ \pm}[z] \nabla^{4}\right) w^{ \pm}-\left(\beta_{1}^{ \pm} \nabla+\beta_{3}^{ \pm}[z] \nabla^{3}+\beta_{5}^{ \pm}[z] \nabla^{5}\right) u^{ \pm}, \\
\Phi[x, z, t] & =\left(1+\alpha_{2}^{ \pm}[z] \nabla^{2}+\alpha_{4}^{ \pm}[z] \nabla^{4}\right) \Phi^{ \pm}+\left(\beta_{1}^{ \pm}+\beta_{3}^{ \pm}[z] \nabla^{2}+\beta_{5}^{ \pm}[z] \nabla^{4}\right) w^{ \pm},
\end{aligned}
$$

where $\left(u^{+}, w^{+}\right)$are the velocities at $z=z_{0}$, and $\left(u^{-}, w^{-}\right)$are the pseudo-velocities at $z=z_{1}$. We choose $z_{1}$ to be midway between the sea bottom $z_{b}=-h$ and $z_{0}$, while $z_{2}$ is introduced to represent half the distance from $z_{0}$ to $z_{b}$. Consequently we get

$$
z_{1}=\frac{1}{2}\left(z_{0}+z_{b}\right), \quad \text { and } \quad z_{2}=\frac{1}{2}\left(z_{0}-z_{b}\right),
$$


while the generalized velocity coefficients read

$$
\begin{array}{lll}
\alpha_{2}^{+}[z]=-\frac{\left(z-z_{0}\right)^{2}}{2}, & \alpha_{2}^{-}[z]=-\frac{\left(z-z_{1}\right)^{2}}{2}+\frac{z_{2}^{2}}{18}, \\
\alpha_{4}^{+}[z]=\frac{\left(z-z_{0}\right)^{4}}{24}, & \alpha_{4}^{-}[z]=\frac{\left(z-z_{1}\right)^{4}}{24}-\frac{z_{2}^{2}\left(z-z_{1}\right)^{2}}{36}+\frac{z_{2}^{4}}{504}, \\
\beta_{1}^{+}[z]=\left(z-z_{0}\right), & \beta_{1}^{-}[z]=\left(z-z_{1}\right), \\
\beta_{3}^{+}[z]=-\frac{\left(z-z_{0}\right)^{3}}{6}, & \beta_{3}^{-}[z]=-\frac{\left(z-z_{1}\right)^{3}}{6}+\frac{z_{2}^{2}\left(z-z_{1}\right)}{18}, \\
\beta_{5}^{+}[z]=\frac{\left(z-z_{0}\right)^{5}}{120}, & \beta_{5}^{-}[z]=\frac{\left(z-z_{1}\right)^{5}}{120}-\frac{z_{2}^{2}\left(z-z_{1}\right)^{3}}{108}+\frac{z_{2}^{4}\left(z-z_{1}\right)}{504} .
\end{array}
$$

The new Flexible-Two-Step formulation simplifies to the original Two-Step formulation for the conventional choice $z_{0}=0$.

7.2. Instability analysis and its numerical verification. In the following, we look for harmonic solutions on the form

$$
u^{ \pm}=\varepsilon B^{ \pm} \cos [k x-\omega t], \quad w^{ \pm}=\varepsilon C^{ \pm} \sin [k x-\omega t], \quad \eta=z_{\text {long }}+\varepsilon A_{0} \cos [k x-\omega t]
$$

and utilize

$$
z_{0} \equiv \sigma h, \quad \text { and } \quad z_{\text {long }} \equiv \delta h .
$$

First, we determine the connection between $C^{+}$and $B^{+}$at $z=z_{0}$ and obtain the result

$$
F_{0}[\lambda]=\lambda\left(\frac{1+\frac{5}{36} \lambda^{2}+\frac{47}{11340} \lambda^{4}+\frac{19}{544320} \lambda^{6}+\frac{1}{15240960} \lambda^{8}}{1+\frac{17}{36} \lambda^{2}+\frac{16}{567} \lambda^{4}+\frac{1}{2240} \lambda^{6}+\frac{29}{15240960} \lambda^{8}+\frac{1}{914457600} \lambda^{10}}\right)
$$

where $\lambda \equiv k\left(z_{0}-z_{b}\right)=k h(1+\sigma)$. This is obviously similar to (52).

Second, we determine the coefficients in the homogeneous problem (22) and find that $m_{11}=1, m_{21}=-g k$, while $\left(m_{12}, m_{22}\right)$ are given by

$$
\begin{aligned}
& m_{12}=-F_{0}[(1+\sigma) \kappa]\left(1+\frac{1}{2} \gamma^{2} \kappa^{2}+\frac{1}{24} \gamma^{4} \kappa^{4}\right)-\left(\gamma \kappa+\frac{1}{6} \gamma^{3} \kappa^{3}+\frac{1}{120} \gamma^{5} \kappa^{5}\right) \\
& m_{22}=\left(1+\frac{1}{2} \gamma^{2} \kappa^{2}+\frac{1}{24} \gamma^{4} \kappa^{4}\right)+F_{0}[(1+\sigma) \kappa]\left(\gamma \kappa+\frac{1}{6} \gamma^{3} \kappa^{3}+\frac{1}{120} \gamma^{5} \kappa^{5}\right)
\end{aligned}
$$

where $\gamma \equiv \delta-\sigma$ and $\kappa \equiv k h$. Obviously, this result is similar to (33)-(34). This directly leads to the determination of the linear celerity $c$ at the level of $z_{\text {long }}$, and we find that $c$ becomes a function of $\sigma, \delta$ and $\kappa$.

Third, the problem of instability is again described by (35) with coefficients given by (36). Figure 12 shows the outcome of the analysis compared to the corresponding numerical results. Results are shown for $\sigma=-0.05$ (top panel) and $\sigma=-0.10$ (bottom panel). Both should be compared to Figure 6 , which was made with $\sigma=0$. We conclude that by pushing down the levels of $\sigma$, we also push down the critical values of $\delta$. In other words, for decreasing $\sigma$ we can accept lower and lower through-levels of the long waves without triggering the trough instabilities. We can also conclude, that if we choose $\sigma$ to be below the lowest occurring trough-level (which corresponds to assuming that $\sigma \leq \delta$ ), 
trough instabilities will never occur in the system. We note that the stability results obtained from the numerical simulations tend to agree with the analytical curves.

Finally, it is of relevance to estimate the relative error of the celerity of the short wave when it travels on the long wave. We define this error by

$$
E \equiv\left(\frac{c^{2}[\sigma, \delta, \kappa]}{g h(1+\delta)}\right) /\left(\frac{\tanh [\kappa(1+\delta)]}{\kappa(1+\delta)}\right) .
$$

Figure 13 shows the result for a fixed value of $\sigma=-0.10$, and for $\delta$ varying within the interval $-0.10 \leq \delta \leq 0.10$ which represents horizontal levels from the trough to the crest of a linear long wave with amplitude $0.10 h$. Obviously, (79) is only a crude estimate of the impact of a long wave on a short wave. A much more comprehensive solution for this problem was given by e.g. Zhang et al. (1993).

\section{Discussion of Older Boussinesq-TyPe formulations}

So far, we have analysed and discussed Boussinesq-type formulations which could be coined fully-nonlinear in the sense that nonlinear dispersive terms are included. Other formulations of this type are e.g. Wei et al. (1995), Madsen \& Schäffer (1998), Gobbi et al. (2000) and Lynett \& Liu (2004), and they all suffer from the trough instability identified and investigated in this work.

As an example, we analyse the formulation by Wei et al. (1995) in the following. On a flat bottom and in a single horizontal dimension this can be written in the form

$$
\begin{aligned}
\frac{\partial \eta}{\partial t}+h \frac{\partial u_{E}}{\partial x}+\frac{\partial\left(\eta u_{E}\right)}{\partial x}+\left(\alpha+\frac{1}{3}\right) h^{3} \frac{\partial^{3} u_{E}}{\partial x^{3}}+\Lambda_{1} & =0 \\
\frac{\partial u_{E}}{\partial t}+g \frac{\partial \eta}{\partial x}+u_{E} \frac{\partial u_{E}}{\partial x}+\alpha h^{2} \frac{\partial^{3} u_{E}}{\partial x^{2} \partial t}+\Lambda_{2} & =0,
\end{aligned}
$$

where the nonlinear dispersive terms are given by

$$
\begin{aligned}
\Lambda_{1}= & \frac{\partial}{\partial x}\left(\left(\alpha h^{2} \eta-\frac{1}{2} h \eta^{2}-\frac{1}{6} \eta^{3}\right) \frac{\partial^{2} u_{E}}{\partial x^{2}}\right), \\
\Lambda_{2}= & \frac{\partial}{\partial x}\left(\left(\alpha h^{2}-h \eta-\frac{1}{2} \eta^{2}\right) u_{E} \frac{\partial^{2} u_{E}}{\partial x^{2}}-\left(h \eta+\frac{1}{2} \eta^{2}\right) \frac{\partial^{2} u_{E}}{\partial x \partial t}\right)+ \\
& \frac{\partial}{\partial x}\left(\frac{1}{2}(h+\eta)^{2} \frac{\partial u_{E}}{\partial x} \frac{\partial u_{E}}{\partial x}\right),
\end{aligned}
$$

and

$$
\alpha \equiv \frac{z_{E}}{h}+\frac{1}{2}\left(\frac{z_{E}}{h}\right)^{2}
$$

The expansion level $z_{E}$ is chosen so that $\alpha=-2 / 5$ by which the linear dispersion relation becomes a Padé $(2,2)$ expansion which is applicable up to $k h=2.34$ based on a threshold of $2 \%$ error compared to the fully dispersive target. We follow the stability procedure from section 4 and obtain (22) with

$$
\begin{aligned}
& m_{12}=-\kappa(1+\delta)+\kappa^{3}\left(\alpha+\frac{1}{3}\right)+\kappa^{3}\left(\alpha \delta-\frac{1}{2} \delta^{2}-\frac{1}{6} \delta^{3}\right), \\
& m_{22}=1-\alpha \kappa^{2}+\kappa^{2}\left(\delta+\frac{1}{2} \delta^{2}\right),
\end{aligned}
$$


while $m_{11}$ and $m_{21}$ are given by (23). The stability analysis follows (35) with (36) and the corresponding zones of instability are shown in Figure 14.

As an alternative, we may consider the formulation by Nwogu (1993) including only linear dispersive terms. This is obtained by setting $\Lambda_{1}=\Lambda_{2}=0$ in (80)-(81). The embedded linear dispersion relation is the same as in Wei et al. (1995) but now the coefficients change to

$$
\begin{aligned}
& m_{12}=-\kappa(1+\delta)+\kappa^{3}\left(\alpha+\frac{1}{3}\right) \\
& m_{22}=1-\alpha \kappa^{2}
\end{aligned}
$$

This formulation contains no trough instabilities, and this is a general conclusion for all Boussinesq formulations based on linear dispersive terms. Examples are e.g. Peregrine (1967), Madsen \& Sørensen (1992) and Nwogu (1993).

\section{TAYLOR FORMULATIONS COMBINED WITH THE EXACT DISPERSION RELATION}

All Boussinesq-type formulations incorporate an approximate linear dispersion relation, and as we have seen from sections $4-8$, the accuracy of this dispersion relation has a significant influence on the potential zones of instability in $(\delta, k h)$-space. It is therefore of interest to combine the Taylor series formulations such as (12)-(14) with the exact relation between $w_{0}$ and $u_{0}$ i.e. utilizing $(20)$ with the connection

$$
F_{0}[\kappa]=\tanh [\kappa] .
$$

This corresponds to embedding the exact linear dispersion relation in the system. We analyse $4 t h$-, 5th- and 6th-order Taylor formulations combined with (89) and conclude that none of them are prone to trough instabilities.

It should be emphasized, that Dommermuth \& Yue (1987) used these Taylor formulations as the starting point for developing their classical high-order-spectral (HOS) method. The key was to invert the relations between the surface variables and the variables at $z=0$ by successive approximations and to derive an explicit expression for $w_{s}$ in terms of $\left(\eta, \Phi_{s}\right)$. By analysis we conclude that the classical HOS formulations do not suffer from trough instabilities.

\section{Summary AND CONCLUSiOns}

In this work, we have analysed Boussinesq-type formulations for instabilities, which may occur in the trough of wave trains in connection with relatively high values of the Nyquist wave number. It can be concluded, that this type of instability will occur if the formulation incorporates nonlinear dispersive terms and if the embedded linear dispersion relation is not exact. This excludes most of the classical Boussinesq formulations, but includes all of the so-called "fully-nonlinear" formulations. The importance of the potential instability depends on the Nyquist wave number $\left(k_{N} h\right)$ and on the level of the deepest trough in the wave train $\left(\eta_{\min } / h\right)$. If these fall into the unstable (gray) regions shown throughout this paper, numerical models will be prone to trough instability. We emphasize that the analysis of trough instabilities performed in this work, focuses directly on the governing equations, and it is not dependent on the time step, the time stepping scheme or the way in which spatial derivatives are numerically approximated.

In section 4, we have analysed two versions of the Boussinesq formulation by Agnon et al. (1999), where the Taylor expansion from $z=0$ to $z=\eta$ is truncated at fourth-order 
and at fifth-order, respectively. Both versions incorporate a Padé $(4,4)$ linear dispersion relation accurate up to approximately $k h=6$. As shown in Figures 4 and 5 , trough instabilities may occur for certain levels of the overall wave troughs specified as $\delta h$, and the trend is that the trouble-zones get closer and closer to the still water level for increasing values of $k_{N} h$. The validity of the analysis is confirmed by numerical calculations and they are in very good agreement.

In section 5, we have analysed the One-Step Padé and the Two-Step Taylor/Padé formulations by Madsen et al. (2002, 2003). Both versions incorporate a linear dispersion relation accurate up to approximately $k h=26$. As shown in Figures 6 and 7, trough instabilities may occur in both formulations, and again the trend is that the trouble-zones get closer and closer to the still water level for increasing values of the Nyquist wave number. It is, however, also obvious that the unstable (gray) zones in Figures 6 and 7 are less pronounced than those in Figure 4 and 5 within the same interval of Nyquist wave numbers. The reason is the much higher accuracy of the embedded linear dispersion relation. Again, we note how the validity of the analysis is confirmed by the numerical calculations.

In section 6, we have analysed the recent multi-layer formulation by Liu et al. (2018). Specifically, we have focused on their one-layer, two-layer, three-layer and four-layer formulations, each incorporating third-order operators. The embedded linear dispersion relations are accurate up to approximately $k h=10,62,277$ and 938, respectively. As shown in Figures 8, 9, 10 and 11, these formulations are also prone to trough instabilities. Again we can conclude that the unstable zones become thinner and less pronounced when the accuracy of the linear dispersion relation is improved.

In section 7, we have shown how the Two-Step Taylor/Padé formulation can be modified to move or remove the instability zone. The idea is to generalize the velocity formulation so that Padé properties are obtained at the sea bed as well as at the arbitrary top level $z_{0}=\sigma h$. In Figure 12, it is shown that by pushing down the level of $\sigma$, we also push down the unstable zone, so that lower and lower trough-levels of the wave train can be accepted without triggering trough instabilities. By choosing $\sigma \leq \delta$ instabilities vanish completely. However, there is a penalty to be paid: The deeper we push $\sigma$, the more inaccurate the linear dispersion relation becomes. This is illustrated in Figure 13. The technique demonstrated in section 7 can easily be incorporated in the other Boussinesq formulations discussed in sections 4-6.

In section 8, we have discussed and analysed two of the popular older Boussinesq formulations from the 1990s: The weakly nonlinear formulation by Nwogu (1993), which includes only linear dispersive terms, and the "fully nonlinear" formulation by Wei et al. (1995), which includes nonlinear dispersive terms. It is found that the fully nonlinear formulation is prone to trough instabilities (see Figure 14), while the weakly nonlinear is not.

In section 9, we briefly discuss the option of combining the nonlinear Taylor formulation with the exact linear dispersion relation. This is relevant, because it forms the starting point of Higher Order Spectral (HOS) perturbation methods as derived by e.g. Dommermuth \& Yue (1987). We conclude that introducing the exact linear dispersion relation removes the problem of trough instability, which is also absent in the HOS formulations.

The present paper has diagnosed a new mechanism which can promote numerical instabilities in numerous modern Boussinesq-type formulations for water waves. This problem has perplexed the authors for some time. We hope the analysis of and the 
solution to this problem may help other researchers who may have encountered similar issues.

\section{REFERENCES}

[1] Agnon, Y., Madsen, P.A. and Schäffer, H.A. 1999 A new approach to high order Boussinesq models. J. Fluid Mech. 399, 319-333.

[2] Chazel, F., Benoit, M., Ern, A. and Piperno, S. 2009 A double-layer Boussinesqtype model for highly nonlinear and dispersive waves. Proc. R. Soc. Lond. A 465, 2319-2346.

[3] Dommermuth, D.G. and Yue, D.K.P. 1987 A high-order spectral method for the study of nonlinear gravity waves. J. Fluid Mech. 184, 267-288.

[4] Gobbi, M.F., Kirby, J.T. and Wei, G. 2000 A fully nonlinear Boussinesq model for surface waves. II. Extension to $\mathrm{O}\left(k h^{4}\right)$. J. Fluid Mech. 405, 182-210.

[5] Kennedy, A.B., Kirby, J.T., Chen, Q. and Dalrymple, R.A. 2001 Boussinesq-type equations with improved nonlinear behaviour. Wave Motion 33, 225-243.

[6] Liu, Z.B. and Fang, K.Z. 2016 A new two-layer Boussinesq model for coastal waves from deep to shallow water: derivation and analysis. Wave Motion 67, 1-14.

[7] Liu, Z.B., Fang, K.Z. and Cheng, Y.Z. 2018 A new multi-layer irrotational Boussinesq-type model for highly nonlinear and dispersive surface waves over a mildly sloping seabed. J. Fluid Mech. 842, 323-353.

[8] Lynett, P. and Liu, P.L.-F. 2004 A two-layer approach to wave modelling. Proc. R. Soc. Lond. A 460, 2637-2669.

[9] Madsen, P.A., Bingham, H.B. and Liu, H. 2002 A new Boussinesq method for fully nonlinear waves from shallow to deep water. J. Fluid Mech. 462, 1-30.

[10] Madsen, P.A., Bingham, H.B. and Schäffer, H.A. 2003 Boussinesq-type formulations for fully nonlinear and extremely dispersive water waves - Derivation and analysis. Proc. Roy. Soc. London, A 459, 1075-1104.

[11] Madsen, P.A. and Agnon, Y. 2003 Accuracy and convergence of velocity formulations for water waves in the framework of Boussinesq theory. J. Fluid Mech. 477, 285-319.

[12] Madsen, P.A. and Schäffer, H.A. 1998 Higher-order Boussinesq-type equations for surface gravity waves: derivation and analysis. Phil. Trans. R. Soc. Lond. A 356, 3123-3181.

[13] Madsen, P.A. and Sørensen, O.R. 1992 A new form of the Boussinesq equations with improved linear dispersion characteristics. Part 2: A slowly varying bathymetry. Coastal Engng 18, 183-204

[14] Nwogu, O. 1993 Alternative form of Boussinesq equations for nearshore wave propagation. J. Wtrwy Port Coastal Ocean Engng 119, 618-638.

[15] Peregrine, D. H. 1967 Long waves on a beach. J. Fluid Mech., 27, 815-827. 
[16] Serre, F. 1953 Contribution a l'etude des ecoulements permanents et variables dans les canaux. La Houille Blanche 3, 374-388.

[17] Wei, G.E., Kirby, J.T., Grilli, S.T. and Subramanya, R. 1995 A fully nonlinear Boussinesq model for surface waves. Part 1. Highly nonlinear unsteady waves. J. Fluid Mech. 294, 71-92.

[18] Witting, J.M. 1984 A unified model for the evolution of nonlinear water waves. J. Comput. Phys. 56, 203-236.

[19] Zakharov, V.E. 1968 Stability of periodic waves of finite amplitude on the surface of a deep fluid. J. Appl. Mech. Tech. Phys. 9, 190-194.

[20] Zhang, J., Hong, K. and Yue, D.K.P. 1993 Effects of wave length ratio on wave modelling. J. Fluid Mech. 248, 107-127. 


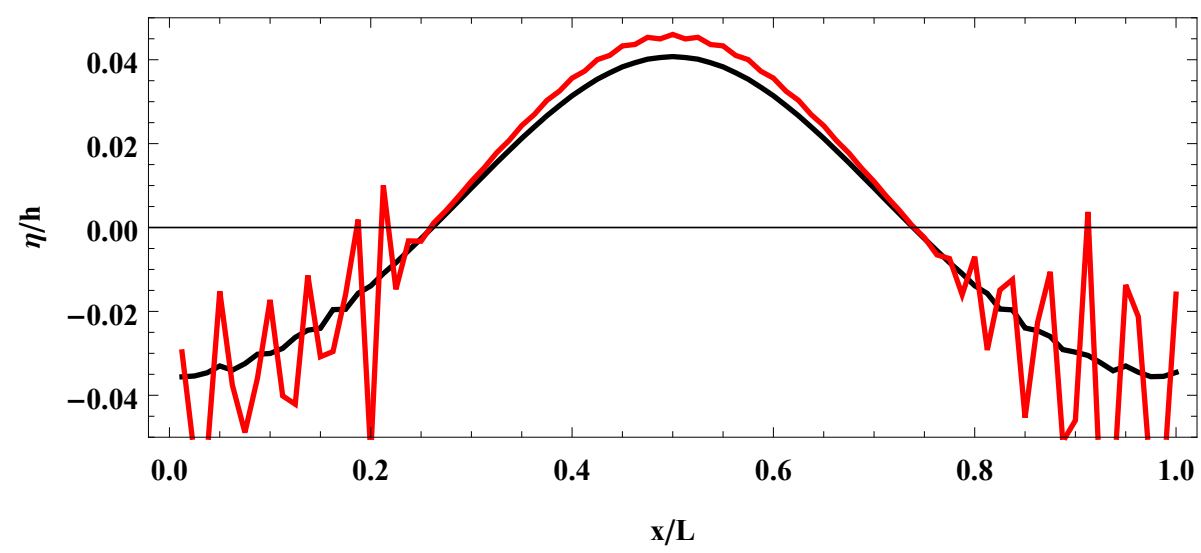

Figure 1. Numerical solution to the formulation by Agnon et al. (1999) with fourthorder operators. Input given by eqs. (28) and (30). Black: Surface elevation at time step $n=89$. Red: Surface elevation at time step $n=95$.

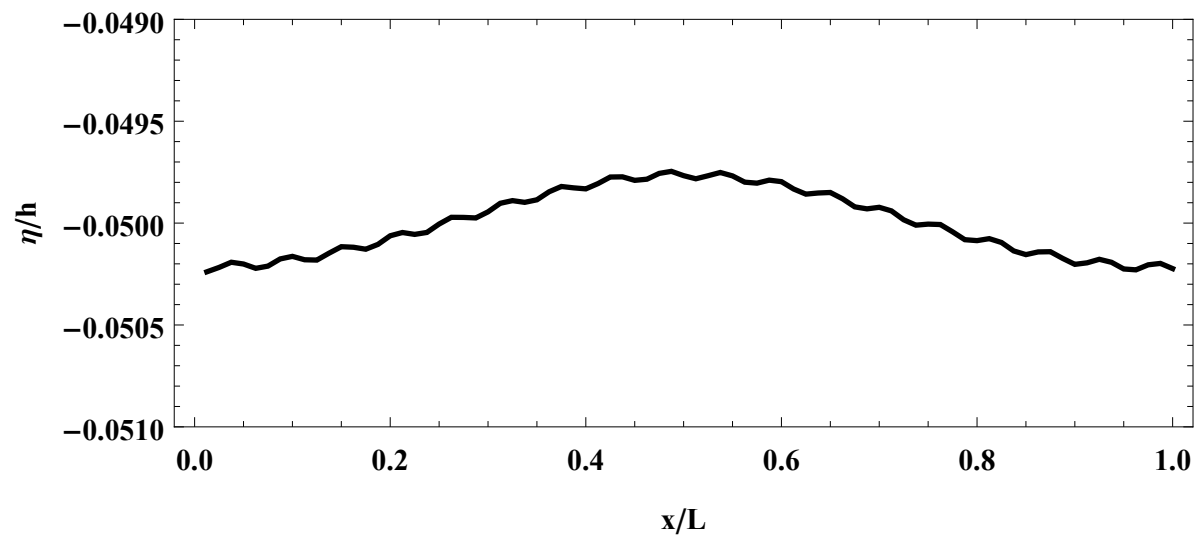

Figure 2. Numerical solution to the formulation by Agnon et al. (1999) with fourthorder operators. Input given by eqs. (31) and (30). Black: Surface elevation at time step $n=60$. 


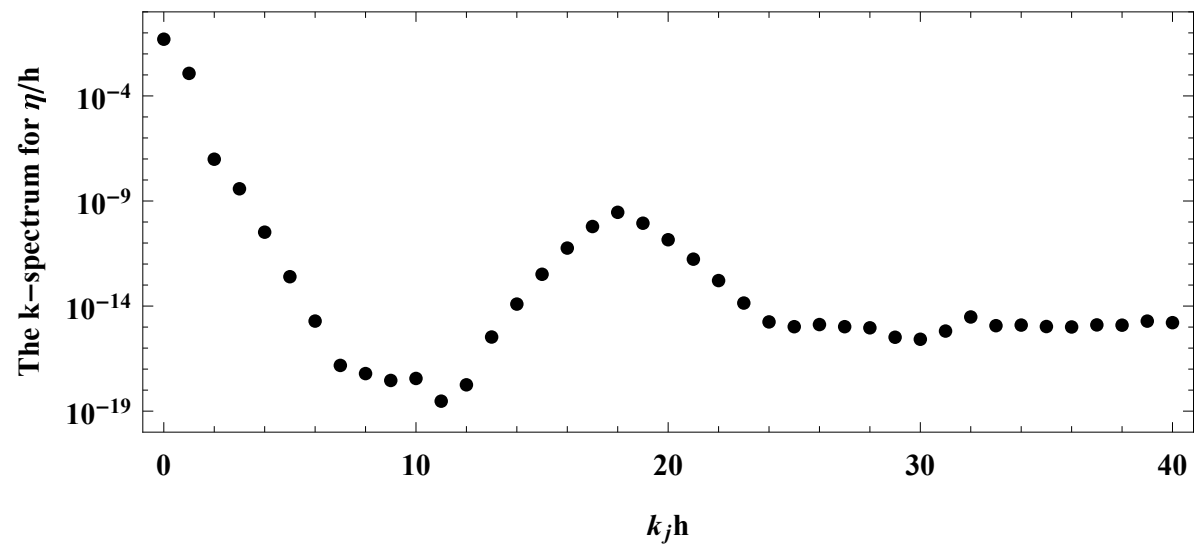

Figure 3. Absolute values of the complex k-spectrum for the surface elevation at time step $\mathrm{n}=40$. Input given by eqs. (31) and (30).

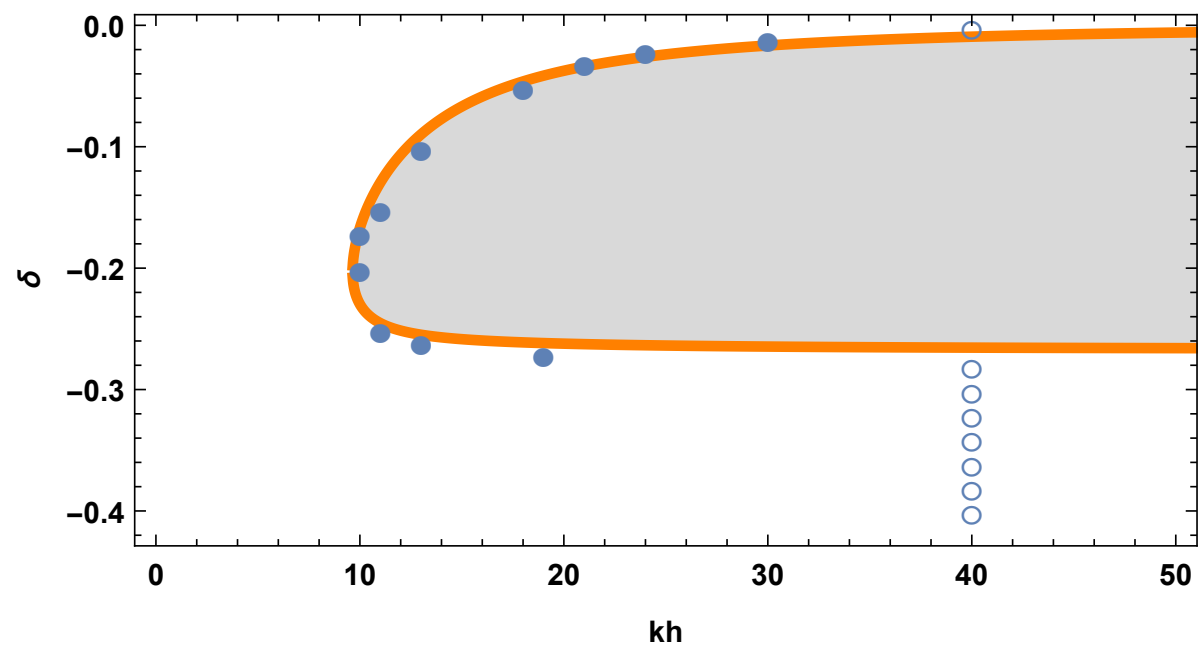

Figure 4. Analysis and verification of the formulation by Agnon et al. (1999) with fourth-order operators. Theoretical zones of instability: 1) Imaginary eigenvalues (grey area); 2) Singularities in the linear celerity (orange fat line). Numerical simulations: Stable (०), Unstable $(\bullet)$. 


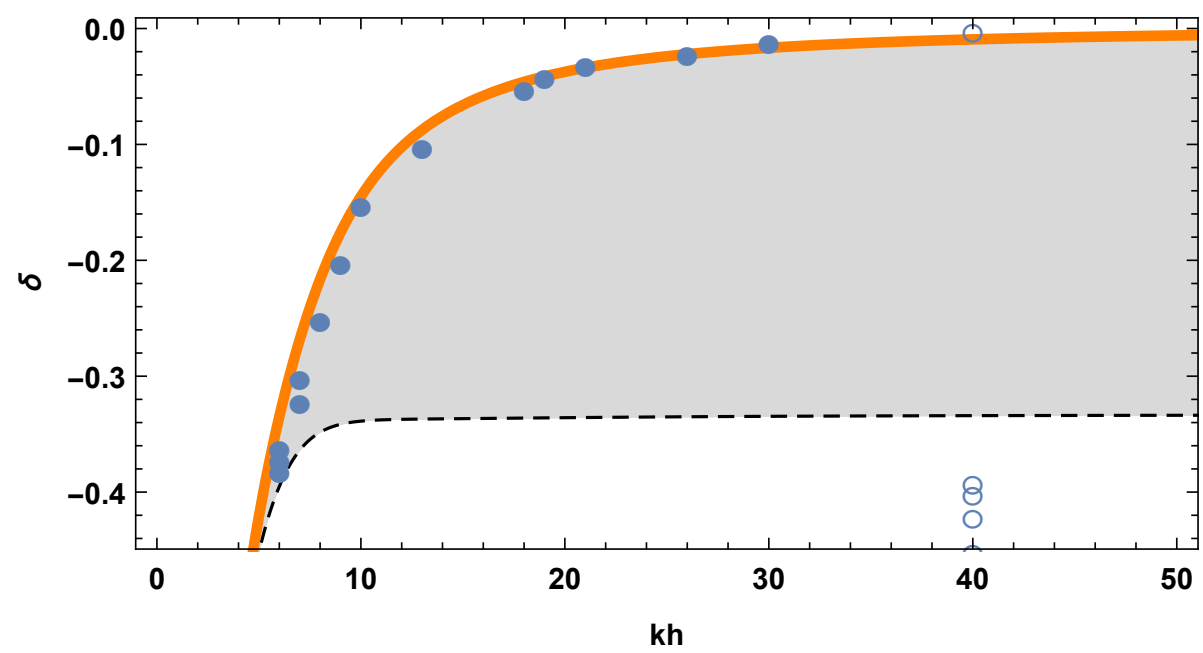

Figure 5. Analysis and verification of the formulation by Agnon et al. (1999) with fifth-order operators. Theoretical zones of instability: 1) Imaginary eigenvalues (grey area); 2) Singularities in the linear celerity (orange fat line). Numerical simulations: Stable (o), Unstable $(\bullet)$.

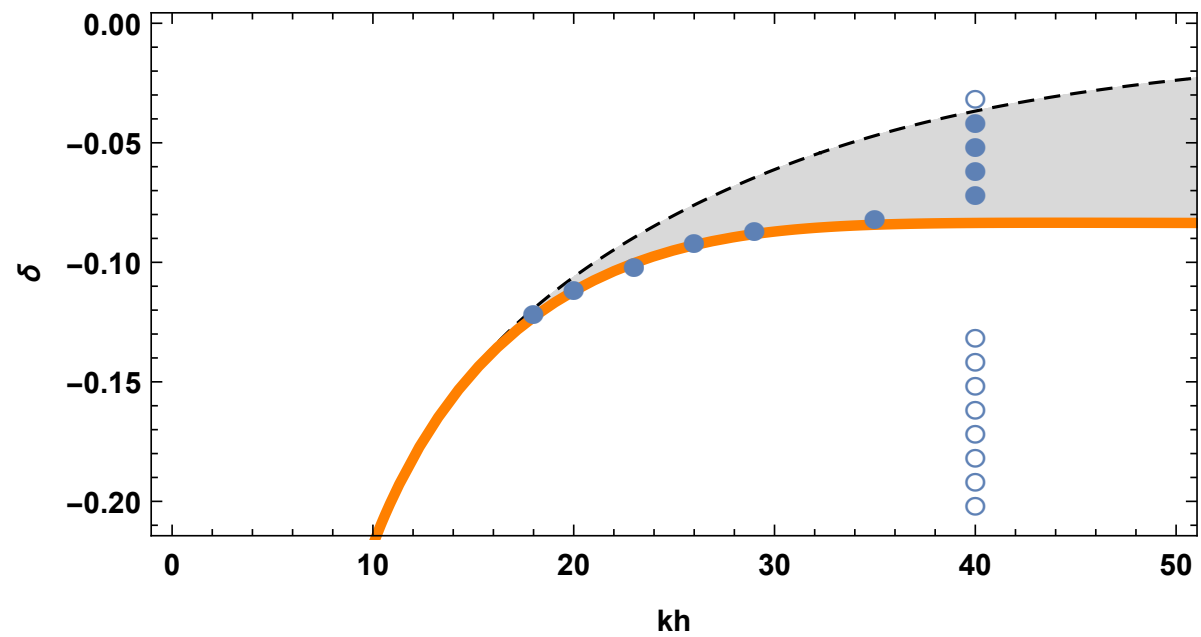

Figure 6. Analysis and verification of the Two-Step Taylor-Pade formulation by Madsen et al. (2002) with fifth-order operators. Theoretical zones of instability: 1) Imaginary eigenvalues (grey area); 2) Singularities in the linear celerity (fat line). Numerical simulations: Stable (o), Unstable (•). 




Figure 7. Analysis and verification of the One-Step Pade formulation by Madsen et al. (2002) with fifth-order operators. Theoretical zones of instability: 1) Imaginary eigenvalues (grey area); 2) Singularities in the linear celerity (fat line). Numerical simulations: Stable (०), Unstable $(\bullet)$.



Figure 8. Analysis and verification of the One-Layer formulation by Liu et al. (2017). Theoretical zones of instability: 1) Imaginary eigenvalues (grey area); 2) Singularities in the linear celerity (fat line). Numerical simulations: Stable (o), Unstable $(\bullet)$. 


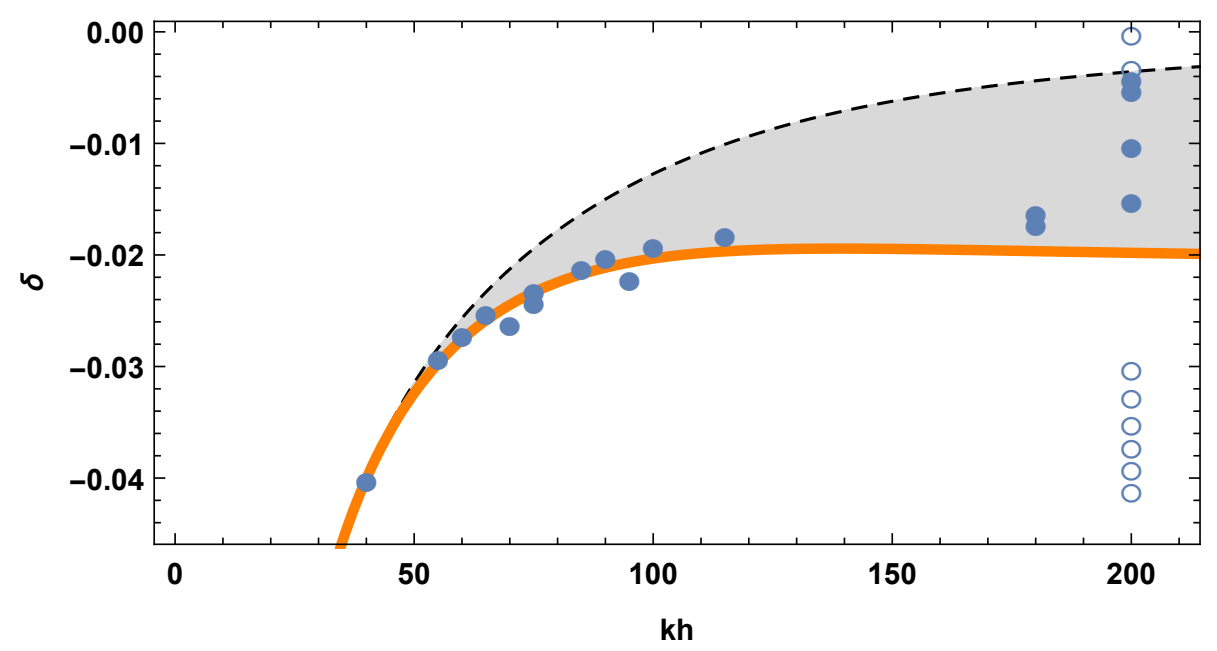

Figure 9. Analysis and verification of the Two-Layer formulation by Liu et al. (2017). Theoretical zones of instability: 1) Imaginary eigenvalues (grey area); 2) Singularities in the linear celerity (fat line). Numerical simulations: Stable (o), Unstable $(\bullet)$.

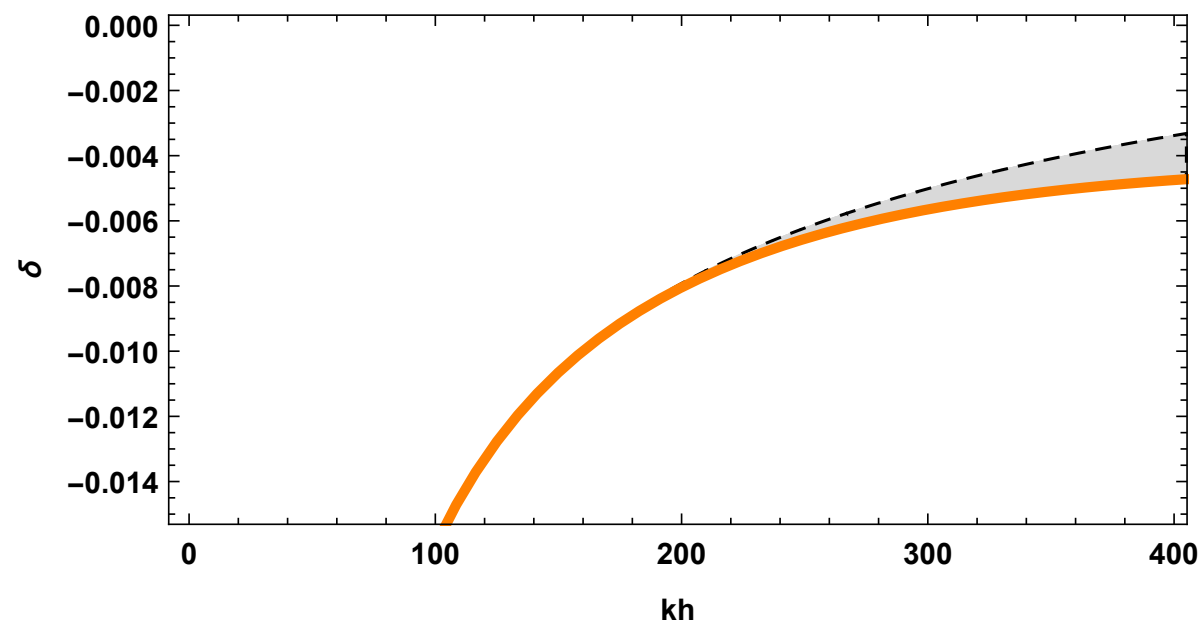

Figure 10. Analysis of the Three-Layer formulation by Liu et al. (2017). Theoretical zones of instability: 1) Imaginary eigenvalues (grey area); 2) Singularities in the linear celerity (fat line). 


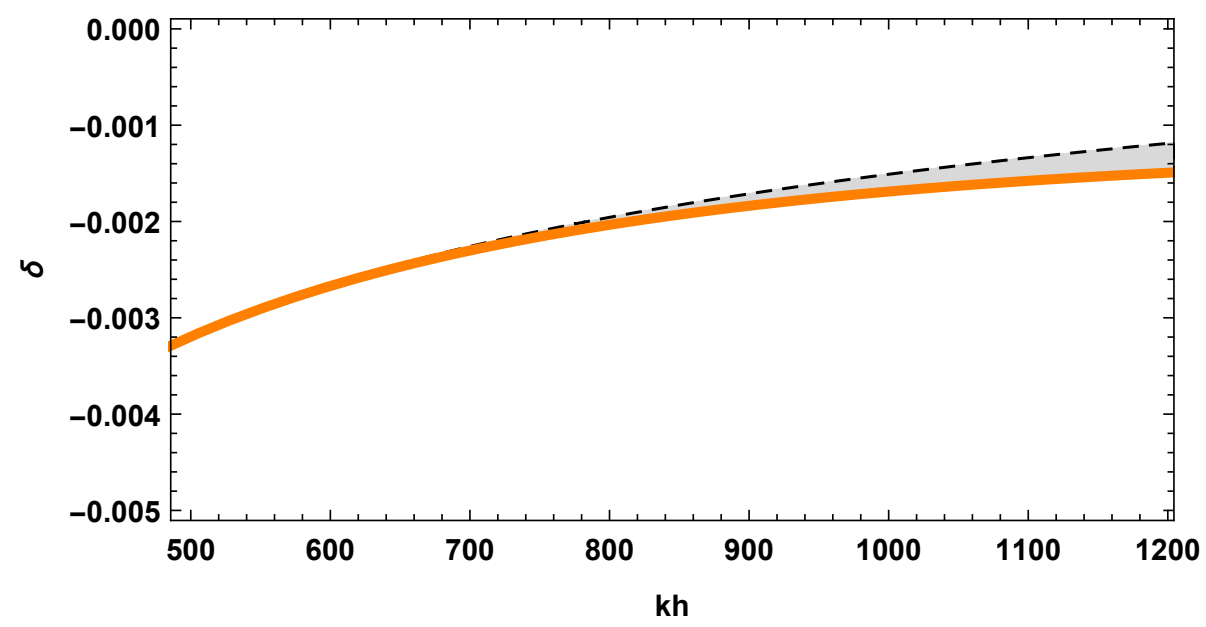

Figure 11. Analysis of the Four-Layer formulation by Liu et al. (2017). Theoretical zones of instability: 1) Imaginary eigenvalues (grey area); 2) Singularities in the linear celerity (fat line). 

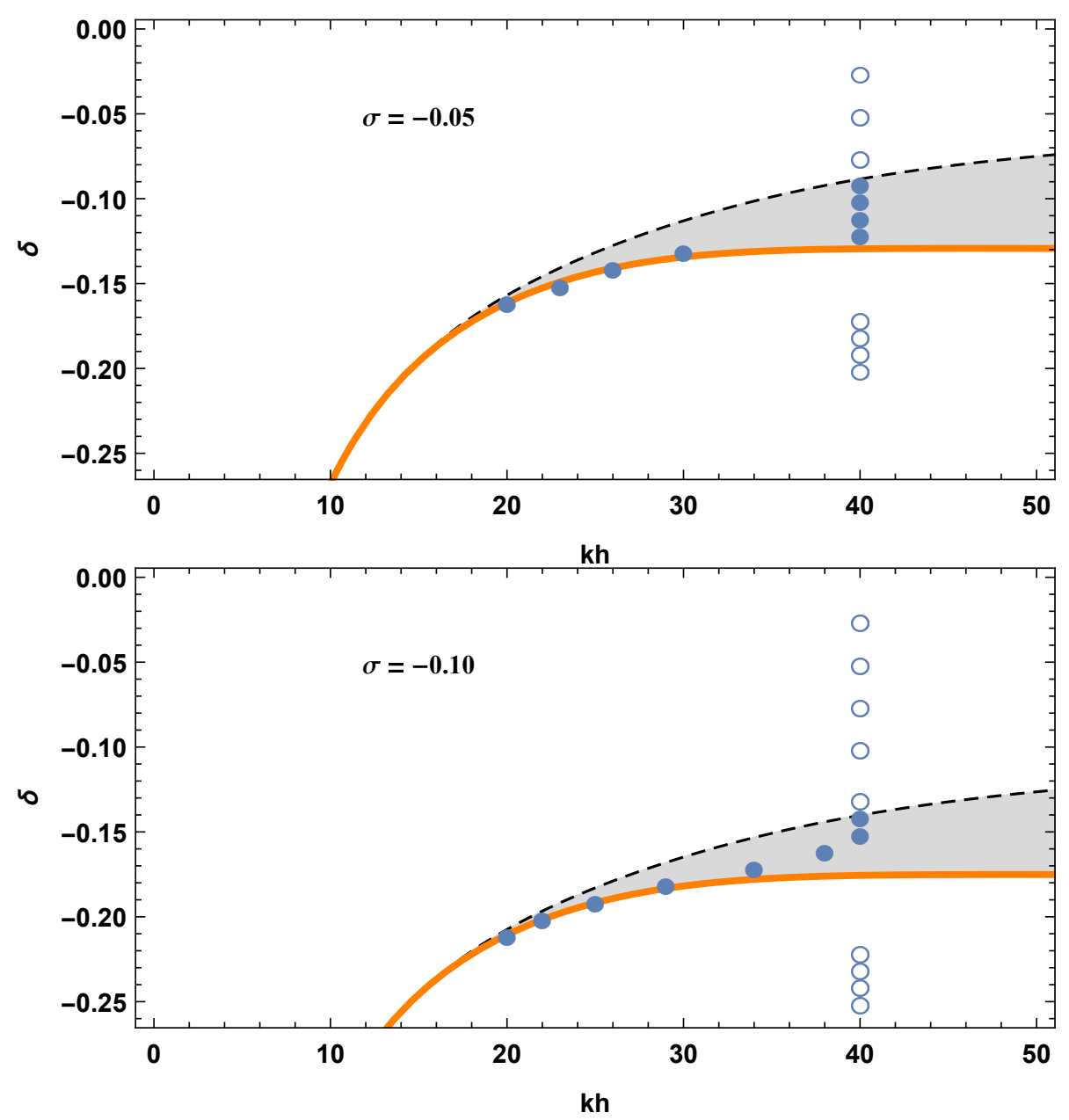

Figure 12. Analysis and verification of the new Flexible-Two-Step Taylor-Pade formulation with fifth-order operators. Top panel: $\sigma=-0.05$; Bottom panel: $\sigma=-0.10$. Theoretical zones of instability: 1) Imaginary eigenvalues (grey area); 2) Singularities in the linear celerity (fat line). Numerical simulations: Stable (o), Unstable $(\bullet)$. 




Figure 13. Analysis of the new Flexible-Two-Step Taylor-Pade formulation with fifth-order operators and $\sigma=-0.10$. Relative accuracy of the celerity: 1) $\delta=$ -0.10 ;2) $\delta=-0.075$; 3) $\delta=-0.05$;4) $\delta=-0.025$; 5) $\delta=0$; 6) $\delta=0.05$; 7) $\delta=0.10$.

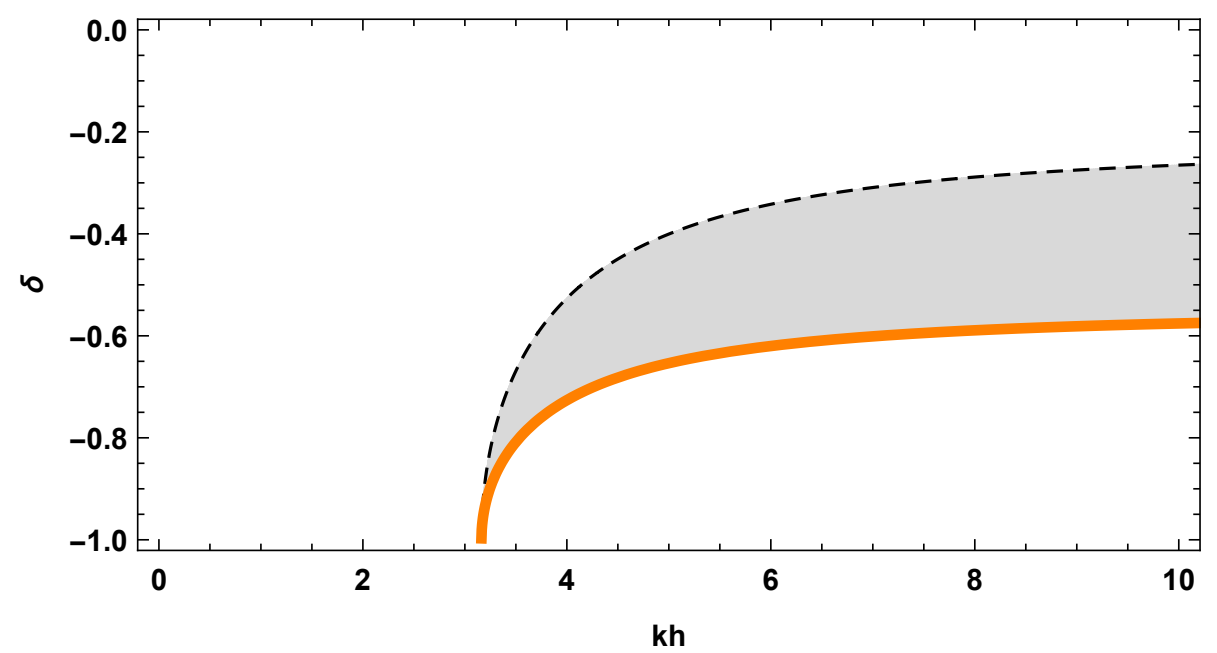

Figure 14. Analysis of the formulation by Wei Kirby (1995). Theoretical zones of instability: 1) Imaginary eigenvalues (grey area); 2) Singularities in the linear celerity (fat line). 